\title{
Molecular dynamics simulations of inverse patchy colloids ${ }^{\star}$
}

\author{
Silvano Ferrari ${ }^{1}$, Gerhard $\mathrm{Kahl}^{1}$, and Emanuela Bianchi ${ }^{1,2, a}$ \\ 1 Institut für Theoretische Physik, TU Wien and Center for Computational Materials Science (CMS), \\ Wiedner Hauptstraße 8-10, A-1040 Wien, Austria \\ 2 Faculty of Physics, University of Vienna, Boltzmanngasse 5, A-1090 Vienna, Austria
}

Received 26 January 2018 and Received in final form 6 March 2018

Published online: 28 March 2018

(C) The Author(s) 2018. This article is published with open access at Springerlink.com

\begin{abstract}
Inverse patchy colloids are patchy particles with differently charged surface regions. In this paper we focus on inverse patchy colloids with two different polar patches and an oppositely charged equatorial belt, and we describe a model and a reliable and efficient numerical algorithm that can be applied to investigate the properties of these particles in molecular dynamics simulations.
\end{abstract}

\section{Introduction}

Colloids with differently charged surface regions can be generally described as charged patchy particles; in order to distinguish them from conventional patchy particles $[1,2]$, they are often referred to as inverse patchy colloids (IPCs) [3]. Similar to conventional patchy colloids, IPCs are characterized by non-isotropic interaction patterns and a reduced bonding valence; IPCs are nonetheless considered as a different class of systems because, while the behavior of conventional patchy systems is dominated only by an orientation-dependent attraction, IPC systems are characterized by a competition between directional attraction and directional repulsion, leading to more complex assembly scenarios. Intensive research activity by a diverse selection of groups (both experimental and theoretical) have recently focused on IPCs in the context of smart materials design [4-9], where colloids with charged surface patterns offer promising (or surprisingly new) opportunities for the self-assembly of target structures with specific properties at the nano- and micro-scale $[10,11]$.

In this contribution we consider an IPC model that has been developed for hard colloids with a heterogeneous surface charge distribution; the model is based on an accurate coarse-graining procedure $[3,12]$ and has been primarily implemented to be studied in Monte Carlo (MC) simulations $[13,14]$. We consider here a suitably adapted version of the model that can be used in Molecular Dynamics (MD) simulations $[15,16]$, and we describe how to set up and to integrate the equations of motions for an ensemble of such particles in MD calculations. The advantage of MD

\footnotetext{
* Contribution to the Topical Issue "Advances in Computational Methods for Soft Matter Systems" edited by Lorenzo Rovigatti, Flavio Romano, John Russo.

a e-mail: emanuela.bianchi@univie.ac.at
}

over MC simulations is the availability of a real time-scale, allowing thus to study dynamic properties via dynamic correlation functions [16]. Moreover, MD simulations allow for a better optimization on GPUs and an easier paralellization of the code with respect to MC algorithms.

We consider here IPCs carrying only two patches, located on the opposite poles of the colloidal particle; in this picture an IPC can be represented as a linear, rigid molecule composed of three units, where the distances between these three entities are fixed. We note that, in general, when using bond constraints only, one can never recover the correct number of degrees of freedom for a linear molecule consisting of more than two atoms [17]. We thus take advantage of an approach presented in ref. [17] and adapt it to our systems. This approach is able to describe the dynamics of rigid polyatomic systems by the motion of a subset of atoms with properly chosen constraints. For the integration of the equations of motion within the velocity Verlet algorithm [18] we use an algorithm, known in literature as RATTLE [19], that is able to describe the evolution of both the positions and the velocities of all entities in a molecule (i.e., in an IPC), that satisfy the internal geometric constraints within any desired accuracy (limited only by the precision of the calculations).

The paper is organized as follows: in sect. 2 we briefly outline the model and in sect. 3 we present the numerical algorithm that allows to integrate the equations of motion of these particles. The manuscript is closed with concluding remarks.

\section{The model}

The model features a hard spherical particle of radius $\sigma_{c}$ carrying two interaction sites, labeled 1 and 2 , located at 


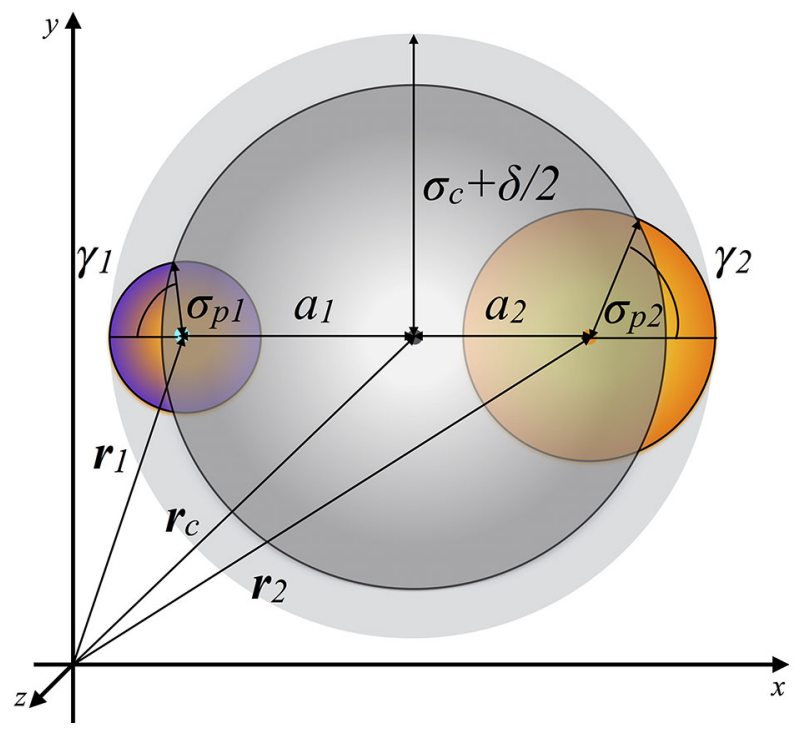

Fig. 1. Representation of an IPC with two different patches in a Cartesian reference system. For the symbols see text.

distances $a_{i}$ (with $i=1,2$ ) from the particle center in opposite direction; such a distance is always smaller than $\sigma_{c}$ so that the sites are located inside the colloid. As a consequence, the corresponding site interaction sphere, with radius $\sigma_{p i}$, extends only partially outside the hard core particle, defining in this way the patch $i$ (see fig. 1). Since all the surface regions of the colloid have the same interaction range, $\delta$, the following relation holds by construction for each patch $i$ (see fig. 1)

$$
\frac{\delta}{2}=a_{i}+\sigma_{p i}-\sigma_{c} .
$$

Since the particle diameter $2 \sigma_{c}$ fixes the unit of length of the model, the parameters to be fixed are $\sigma_{p i}$ and $a_{i}$. The choice of these two parameters defines the colloid interaction range and the patch surface extension, given by the half opening angle $\gamma_{i}$ according to (see fig. 1)

$$
\cos \gamma_{i}=\frac{\sigma_{c}^{2}+a_{i}^{2}-\sigma_{p i}^{2}}{2 a_{i} \sigma_{c}}
$$

The geometric parameters can be fixed to reproduce both the experimental value of $\delta$, which is determined by the electrostatic screening of the surrounding solvent, and the experimental patch sizes, which are related to the surface features of IPCs synthesized in the lab [5].

The pair potential between two IPCs at distance $r$ with a mutual orientation $\Omega$ is given by

$$
U(r, \Omega)= \begin{cases}\infty, & \text { if } r<2 \sigma_{c}, \\ \sum_{\alpha \beta} u_{\alpha \beta} w_{\alpha \beta}(r, \Omega), & \text { if } 2 \sigma_{c}<r<2 \sigma_{\mathrm{c}}+\delta, \\ 0, & \text { if } r_{i j} \geq 2 \sigma_{c}+\delta\end{cases}
$$

where $\alpha$ and $\beta$ specify either a patch or the bare colloid of the first and second IPC, respectively, while $w_{\alpha \beta}$ and

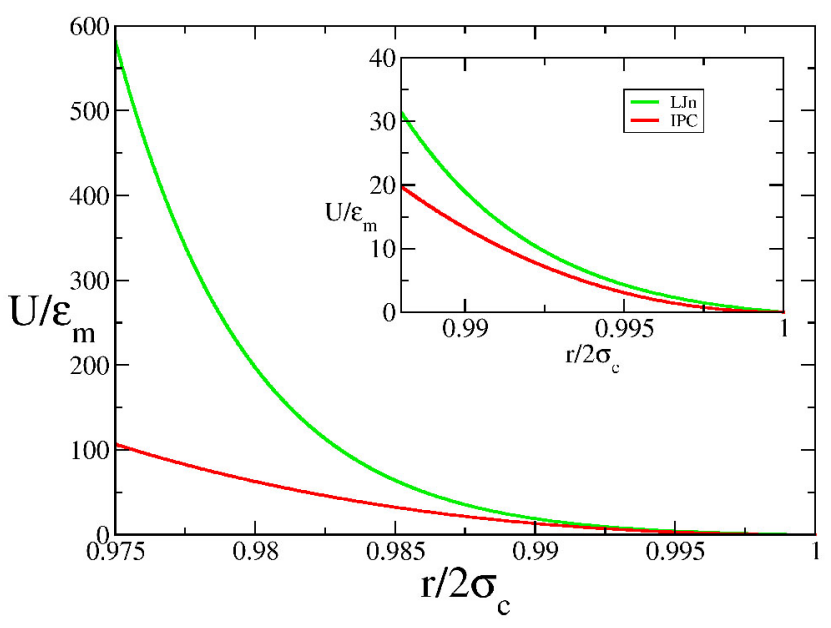

Fig. 2. IPC soft core interaction as specified in eq. (4) (red line) compared to a generalized Lennard-Jones $2 n-n$ potential with $n=100$ (green line) [20].

$u_{\alpha \beta}$ are the geometric weight factor and the characteristic energy strength of the $\alpha \beta$ interaction, respectively. We note that, while the $u_{\alpha \beta}$ are constant values, the $w_{\alpha \beta}$ -as well as the potential $U$ itself- depend on both the inter-particle distance and the relative orientation of the two IPCs. The energy constants $u_{\alpha \beta}$ can be fixed for a precise microscopic system by taking advantage of the analytic description of the model within the Debye-Hückel approach [3,12].

In view of the numerical algorithm that solves the equations of motion of our IPCs within a MD framework (see sect. 3) an IPC can be considered as a linear, rigid molecule, consisting of three entities: the central colloid plus the two interaction spheres, representing the patches.

In order to integrate the equations of motion, we replace the hard core repulsion specified in eq. (3) with a continuous, suitably harsh repulsive soft sphere interaction given by

$$
U(r)=A\left[\left(\frac{2 \sigma_{c}}{r}\right)^{2 k}-2\left(\frac{2 \sigma_{c}}{r}\right)^{k}+1\right] \Theta\left(2 \sigma_{c}-r\right)
$$

with $k=15$ and $A=500 \epsilon_{m}$, where $\epsilon_{m}$ is the energy minimum, obtained when two IPC particles at contact are in a T-shape configuration [3]; $\epsilon_{m}$ sets the unit of energy. This soft core has a deceivingly small exponent compared to the potentials used in, e.g., [20], but the very large $A$ factor contributes in making the potential very stiff (see fig. 2). We note that in our MD simulations particles never get closer to each other than 0.975 (in units of the particle diameter). We checked the consistency between the continuous and the hard core [3] versions of the model by comparing energies, pair distribution functions and static structure factors at several state points with those obtained via Monte Carlo simulations of the original model. No significant differences were observed (see fig. 3). 


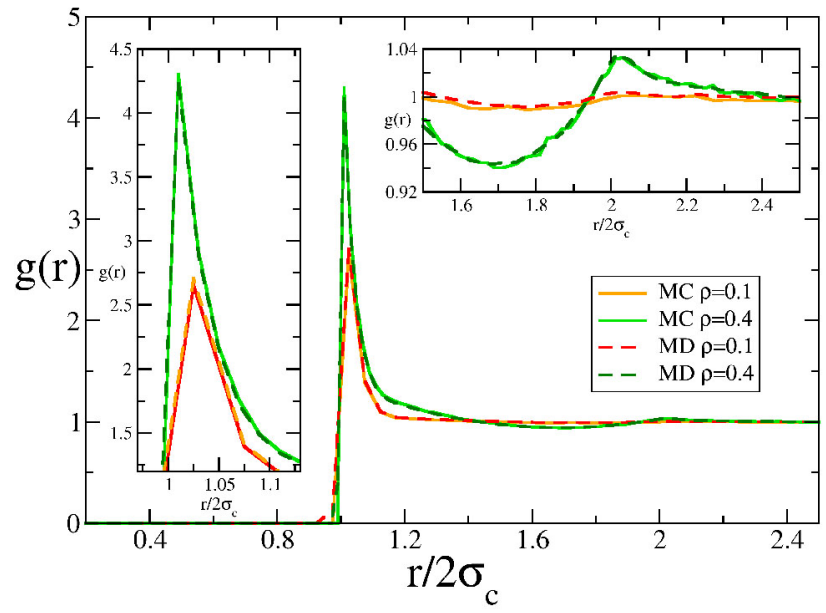

Fig. 3. Monte Carlo (MC) results for the hard core potential reproduce Molecular Dynamics (MD) results for the harshly repulsive soft potential: the radial distribution functions in the fluid phase for an IPC system usually referred to as $30 n[13$, 15] are shown at two state points, namely both points are at $T=0.3$, while $\rho=0.1$ and $\rho=0.4$. The main panel shows the full $g(r)$ as a function of the inter-particle distance, the vertical inset shows an enlargement around the first peak, the horizontal inset zooms over the second peak. The MD results are obtained from a simulation in the microcanonical ensemble, hence with $T$ we refer to the average temperature after equilibration [16].

\section{The numerical algorithm}

We first set up the equations of motion of the IPCs following the procedure presented in ref. [17] (see sect. 3.1) and then integrate these equations using an algorithm that guarantees that the coordinates and velocities of the entities within a molecule satisfy the internal geometric constraints at each time step [19] (see sect. 3.2).

\subsection{Construction of the equations of motion}

We start from the equations of motion for all the units of our linear molecule, i.e.,

$$
\left\{\begin{array}{l}
m_{c} \ddot{\boldsymbol{r}}_{c}=\boldsymbol{F}_{c}, \\
m_{1} \ddot{\boldsymbol{r}}_{1}=\boldsymbol{F}_{1}, \\
m_{2} \ddot{\boldsymbol{r}}_{2}=\boldsymbol{F}_{2},
\end{array}\right.
$$

where $c$ refers to the colloid center, while 1 and 2 denote the patches. We now want to write these equations so that the internal constraints of our molecule are automatically satisfied. To do so, we consider the patches as the so-called primary particles [17] and we infer the position of the colloid center through

$$
\boldsymbol{r}_{c}=\left(a_{2} / d\right) \boldsymbol{r}_{1}+\left(a_{1} / d\right) \boldsymbol{r}_{2},
$$

where $a_{1}, a_{2}$ are the distances of the patches from the colloid center and $d=a_{1}+a_{2}$. As a consequence of our choice, the constraints which guarantee that the internal architecture of our molecule is preserved are on the position of the colloid center and on the distance between the patches; these conditions can be written in the following form

$$
\left\{\begin{array}{l}
\sigma=\left(\boldsymbol{r}_{1}-\boldsymbol{r}_{2}\right)^{2}-d^{2}=0 \\
\boldsymbol{\tau}=-\boldsymbol{r}_{c}+\left(a_{1} / d\right) \boldsymbol{r}_{2}+\left(a_{2} / d\right) \boldsymbol{r}_{1}=0 .
\end{array}\right.
$$

The equations of motion (5) are then corrected by subtracting the gradient of the constraints multiplied by the Lagrange multipliers $\boldsymbol{\mu}$ and $\lambda$

$$
m_{i} \boldsymbol{r}_{i}=\boldsymbol{F}_{i}-\nabla_{i}(\boldsymbol{\mu} \cdot \boldsymbol{\tau}+\lambda \sigma)
$$

where $i=1,2, c$. Substituting the constraints (7) in (8) we obtain

$$
\left\{\begin{array}{l}
m_{c} \ddot{\boldsymbol{r}}_{c}=\boldsymbol{F}_{c}+\boldsymbol{\mu} \\
m_{1} \ddot{\boldsymbol{r}}_{1}=\boldsymbol{F}_{1}-\left(a_{2} / d\right) \boldsymbol{\mu}-\lambda \boldsymbol{r}_{12} \\
m_{2} \ddot{\boldsymbol{r}}_{2}=\boldsymbol{F}_{2}-\left(a_{1} / d\right) \boldsymbol{\mu}+\lambda \boldsymbol{r}_{12}
\end{array}\right.
$$

where $\boldsymbol{r}_{12}=\boldsymbol{r}_{1}-\boldsymbol{r}_{2}$.

Using the second derivative of the equation for $\tau$ in (7) within the first equation of (9) we obtain the following expression for $\boldsymbol{\mu}$

$$
\boldsymbol{\mu}=-\frac{d^{2}}{I m_{c}} \boldsymbol{F}_{c}+\frac{d a_{1}}{I m_{2}} \boldsymbol{F}_{2}+\frac{d a_{2}}{I m_{1}} \boldsymbol{F}_{1}-\lambda\left[\frac{d a_{2}}{I m_{1}}-\frac{d a_{1}}{I m_{2}}\right] \boldsymbol{r}_{12},
$$

where

$$
I=\frac{d^{2}}{m_{c}}+\frac{a_{1}^{2}}{m_{1}}+\frac{a_{2}^{2}}{m_{2}} .
$$

Since the choice of $\boldsymbol{\mu}$ ensures that the constraint $\boldsymbol{\tau}=0$ is always satisfied, eq. (6) can be used as to calculate the trajectory of the colloid center $\boldsymbol{r}_{c}(t)$ once the trajectories of the patches $\boldsymbol{r}_{1}(t)$ and $\boldsymbol{r}_{2}(t)$ are known. This represents a considerable simplification, since we just need to evaluate a linear algebraic equation in place of solving a secondorder differential equation.

The trajectories of the patches that must be integrated with respect to time can be obtained by substituting the expression for $\boldsymbol{\mu}$ - eq. (10) - into the second and third equations of (9). The final system of equations that must be considered is thus

$$
\left\{\begin{aligned}
& \boldsymbol{r}_{c}=\left(a_{1} / d\right) \boldsymbol{r}_{2}+\left(a_{2} / d\right) \boldsymbol{r}_{1}, \\
& m_{1} \ddot{\boldsymbol{r}}_{1}=\left(1-\frac{a_{2}^{2}}{I m_{1}}\right) \boldsymbol{F}_{1}+\left(-\frac{a_{1} a_{2}}{I m_{2}}\right) \boldsymbol{F}_{2}+\left(\frac{d a_{2}}{I m_{c}}\right) \boldsymbol{F}_{c} \\
&-\lambda\left[1+\frac{a_{1}}{I}\left(\frac{a_{2}}{m_{1}}-\frac{a_{1}}{m_{2}}\right)\right] \boldsymbol{r}_{12}, \\
& m_{2} \ddot{\boldsymbol{r}}_{2}=\left(1-\frac{a_{1}^{2}}{I m_{2}}\right) \boldsymbol{F}_{2}+\left(-\frac{a_{1} a_{2}}{I m_{1}}\right) \boldsymbol{F}_{2}+\left(\frac{d a_{1}}{I m_{c}}\right) \boldsymbol{F}_{c} \\
&+\lambda\left[1+\frac{a_{2}}{I}\left(\frac{a_{2}}{m_{1}}-\frac{a_{1}}{m_{2}}\right)\right] \boldsymbol{r}_{12} .
\end{aligned}\right.
$$




\subsection{Integration of the equations of motion}

The system (12) of coupled differential equations still contains the Lagrange multiplier $\lambda$. A naive substitution of the second and third equations of (12) into the second derivative of the equation for $\sigma$ in (7) yields a solution subject to a large error when discretized on a computer [19]. To cope with this problem an alternative algorithm has been proposed, called RATTLE: the idea behind this concept is to determine the value of $\lambda$ at each numerical integration step, so that $\sigma=0$ is always satisfied within numerical accuracy [19].

We can rewrite the equations of motion for the patches of a single IPC — see eq. (12) — in the following, more compact way

$$
\left\{\begin{array}{l}
m_{1} \ddot{\boldsymbol{r}}_{1}=\tilde{\boldsymbol{F}}_{1}-\alpha_{1} \lambda \boldsymbol{r}_{12} \\
m_{2} \ddot{\boldsymbol{r}}_{2}=\tilde{\boldsymbol{F}}_{2}+\alpha_{2} \lambda \boldsymbol{r}_{12}
\end{array}\right.
$$

where we have defined the effective total forces $\tilde{\boldsymbol{F}}_{1}$ and $\tilde{\boldsymbol{F}}_{2}$; further we have introduced two coefficients $\alpha_{1}$ and $\alpha_{2}$, defined as

$$
\alpha_{1}=1+\frac{a_{2}}{I}\left(\frac{a_{2}}{m_{1}}-\frac{a_{1}}{m_{2}}\right)
$$

and

$$
\alpha_{2}=1+\frac{a_{1}}{I}\left(\frac{a_{2}}{m_{1}}-\frac{a_{1}}{m_{2}}\right) .
$$

In the simulation we compute the real forces between two IPCs by adding up the forces originating from the three individual entities of each IPC. From the real forces we then obtain the effective forces via

$$
\left\{\begin{array}{l}
\tilde{\boldsymbol{F}}_{1}=\left(1-\frac{a_{2}^{2}}{I m_{1}}\right) \boldsymbol{F}_{1}+\left(-\frac{a_{1} a_{2}}{I m_{2}}\right) \boldsymbol{F}_{2}+\left(\frac{d a_{2}}{I m_{c}}\right) \boldsymbol{F}_{c} \\
\tilde{\boldsymbol{F}}_{2}=\left(1-\frac{a_{1}^{2}}{I m_{2}}\right) \boldsymbol{F}_{2}+\left(-\frac{a_{1} a_{2}}{I m_{1}}\right) \boldsymbol{F}_{2}+\left(\frac{d a_{1}}{I m_{c}}\right) \boldsymbol{F}_{c} .
\end{array}\right.
$$

Within the velocity Verlet scheme [18], the differential equations (13) are discretized as

$$
\left\{\begin{aligned}
\boldsymbol{r}_{1}(t+h)= & \boldsymbol{r}_{1}(t)+h \boldsymbol{v}_{1}(t)+\frac{h^{2}}{2 m_{1}} \tilde{\boldsymbol{F}}_{1}(t)-\alpha_{1} \lambda_{r} \boldsymbol{r}_{12}(t) \\
\boldsymbol{r}_{2}(t+h)= & \boldsymbol{r}_{2}(t)+h \boldsymbol{v}_{2}(t)+\frac{h^{2}}{2 m_{2}} \tilde{\boldsymbol{F}}_{2}(t)+\alpha_{2} \lambda_{r} \boldsymbol{r}_{12}(t) \\
\boldsymbol{v}_{1}(t+h)= & \boldsymbol{v}_{1}(t)+\frac{h}{2 m_{1}}\left(\tilde{\boldsymbol{F}}_{i}(t)+\tilde{\boldsymbol{F}}_{i}(t+h)\right) \\
& -\alpha_{1} \lambda_{v} \boldsymbol{r}_{12}(t+h) \\
\boldsymbol{v}_{2}(t+h)= & \boldsymbol{v}_{2}(t)+\frac{h}{2 m_{2}}\left(\tilde{\boldsymbol{F}}_{i}(t)+\tilde{\boldsymbol{F}}_{i}(t+h)\right) \\
& +\alpha_{2} \lambda_{v} \boldsymbol{r}_{12}(t+h)
\end{aligned}\right.
$$

where the $\boldsymbol{v}_{i}(t)$ are the velocities of the patches, $h$ is the time increment and $\lambda_{r}$ and $\lambda_{v}$ are Lagrange multipliers [19].
To solve the equations for the positions of the patches we define

$$
\boldsymbol{s}_{i}(t)=\boldsymbol{r}_{i}(t)+h \boldsymbol{v}_{i}(t)+\frac{h^{2}}{2 m_{i}} \tilde{\boldsymbol{F}}_{i}(t),
$$

with $i=1,2$. The constraint $\sigma=0$ can be thus written as

$$
\left|\boldsymbol{s}_{1}-\alpha_{1} \lambda_{r} \boldsymbol{r}_{12}-\boldsymbol{s}_{2}-\alpha_{2} \lambda_{r} \boldsymbol{r}_{12}\right|^{2}=d^{2},
$$

which is solved with respect to $\lambda_{r}$ giving

$$
\lambda_{r}=\frac{\boldsymbol{s}_{12}^{2}-d^{2}}{2\left(\alpha_{1}+\alpha_{2}\right) \boldsymbol{s}_{12} \cdot \boldsymbol{r}_{12}}+O\left(\lambda_{r}^{2}\right),
$$

where $s_{12}=s_{1}-s_{2}$. By substituting this expression for $\lambda_{r}$ into the first two equations of (17), we calculate the new positions $\boldsymbol{r}_{i}(t+h)$ for the patches of our IPC.

Since terms of order $O\left(\lambda_{r}^{2}\right)$ have been neglected in eq. (20), the patch positions might have to be calculated in an iterative fashion: $\lambda_{r}$ is computed from eq. (20) using the just updated positions until

$$
|| \boldsymbol{r}_{1}(t+h)-\left.\boldsymbol{r}_{2}(t+h)\right|^{2}-d^{2} \mid<10^{-12} d^{2}
$$

is satisfied. In our choice of units, $10^{-12} d^{2}$ is close to the machine epsilon ${ }^{1}$; in fact, $10^{-12}$ was chosen as the smallest number which never caused a convergence problem.

Once the new positions of all patches of all IPCs have been corrected, their velocities are also updated in the following three steps. First, we calculate

$$
\boldsymbol{v}_{i}(t+h / 2)=\boldsymbol{v}_{i}(t)+\frac{h}{2 m_{i}} \tilde{\boldsymbol{F}}_{i}(t) \quad i=1,2 .
$$

In this way, the old forces are no longer needed and their memory space can be used to store the new forces $\tilde{\boldsymbol{F}}_{i}(t+h)$, that are now computed from the new positions $\boldsymbol{r}_{i}(t+h)$. Successively, the velocities are again updated as

$$
\boldsymbol{v}_{i}^{*}(t+h)=\boldsymbol{v}_{i}(t+h / 2)+\frac{h}{2 m_{i}} \tilde{\boldsymbol{F}}_{i}(t+h),
$$

where the $*$ indicates that we still have to correct for the constraints. According to the RATTLE scheme [19], we must enforce the time derivative of the constraint $\left|\boldsymbol{r}_{12}(t+h)\right|^{2}=d^{2}$, i.e.,

$$
\boldsymbol{r}_{12}(t+h) \cdot \boldsymbol{v}_{12}(t+h)=0,
$$

where $\boldsymbol{v}_{12}=\boldsymbol{v}_{1}-\boldsymbol{v}_{2}$. This constraint ensures that the velocities of the patches are not able to stretch or compress the IPC but can only lead to a rotational or translational motion of the particle. Finally, the updated velocities can be calculated by

$$
\boldsymbol{v}_{1}(t+h)=\boldsymbol{v}_{1}^{*}(t+h)-\alpha_{1} \lambda_{v} \boldsymbol{r}_{12}(t+h)
$$

and

$$
\boldsymbol{v}_{2}(t+h)=\boldsymbol{v}_{2}^{*}(t+h)+\alpha_{2} \lambda_{v} \boldsymbol{r}_{12}(t+h) .
$$

1 The machine epsilon $\epsilon$ is the smallest number satisfying $1+\epsilon \neq 1$ in machine algebra. Using 8 bytes variables it is $2^{-53} \simeq 1.11 \cdots \times 10^{-16}$. 
where $\lambda_{v}$ is determined by substituting relations and (26) into the constraint (24), i.e.,

$$
\left[\boldsymbol{v}_{12}^{*}(t+h)-\lambda_{v}\left(\alpha_{1}+\alpha_{2}\right) \boldsymbol{r}_{12}(t+h)\right] \cdot \boldsymbol{r}_{12}(t+h)=0 ;
$$

this relation can be solved (exactly) for $\lambda_{v}$

$$
\lambda_{v}=\frac{\boldsymbol{v}_{12}^{*} \cdot \boldsymbol{r}_{12}}{\left(\alpha_{1}+\alpha_{2}\right) d^{2}} .
$$

We note that, in contrast to eq. (20) for $\lambda_{r}$, eq. (24) for $\lambda_{v}$ is exact, so corrections on $\lambda_{v}$ are taken only if $\lambda_{v}>10^{-12}$ (see the online sample codes of ref. [21]); in this case the correction does not need to be iterative.

The error on a single RATTLE trajectory step is of order $h^{2}$, the same as the unconstrained velocity Verlet algorithm. Thus, there is no loss of precision by using RATTLE, as long as the tolerance on the constrains is small enough. This is proven in appendix B of the RATTLE original paper [19].

\section{Conclusions}

In this contirbution we have presented in detail a numerical approach to set up the equations of motion of an ensemble of colloidal particles with heterogeneously charged surfaces and to solve these equations numerically (within the velocity Verlet scheme) in a molecular dynamics simulation code.

We have focused on the special case where the two charged patches are located on the poles of an oppositely charged colloid. Due to some emerging singularities (that are imposed by the linear internal architecture of the particle) this two-patch model represents a rather challenging case. Within our framework the decorated particle is viewed as a rigid, linear molecule that consists of three entities: the central colloidal particle and the two patches. Following an explicit recommendation in ref. [21], we have refrained in our particular problem from an implementation using quaternions. Our formulation of the equations of motion (which avoids the aforementioned singularities) has been combined with a velocity Verlet integration scheme and can be readily and efficiently implemented in a simulation code, leading to robust and reliable numerical solutions of the equations of motion.

Our focus on the two-patch model gives credit to very recent experimental progress in the synthesis of the such particles in the lab [5] and to their remarkable propensity to self-organize into very unusual, sometimes even exotic structures: examples are intricate translationally and orientationally ordered particle arrangements or complex lamellar structures in three dimensions, which keep their stability over a remarkably broad temperature range $[14,16]$.

Our approach sets the stage for future extensions towards multi-patch models, as they are expected to be synthesized in the lab in the near future. From the conceptual point of view, the non-linear internal structure of a multi-patch particle is considerably easier to handle than a linear patch arrangement. However, the number of constraints imposed by the rigidity of the internal structure implies $n_{p}\left(n_{p}-1\right) / 2$ geometric constraints, $n_{p}$ being the number of patches.

Open Access funding provided by University of Vienna. Financial support by the Austrian Science Fund (FWF) under Proj. Nos. V249-N27 (EB), P23910-N16 (SF) and F41 - SFB ViCoM (GK) is gratefully acknowledged. Computer time at the Vienna Scientific Cluster (VSC) is also gratefully acknowledged.

\section{Author contribution statement}

All the authors were involved in the preparation of the manuscript. All the authors have read and approved the final manuscript.

Open Access This is an open access article distributed under the terms of the Creative Commons Attribution License (http://creativecommons.org/licenses/by/4.0), which permits unrestricted use, distribution, and reproduction in any medium, provided the original work is properly cited.

\section{References}

1. A. Pawar, I. Kretzschmar, Macromol. Rapid Commun. 31, 150 (2010).

2. E. Bianchi, R. Blaak, C.N. Likos, Phys. Chem. Chem. Phys. 13, 6397 (2011).

3. E. Bianchi, G. Kahl, C.N. Likos, Soft Matter 7, 8313 (2011).

4. C. Yigit, J. Heyda, M. Ballauff, J. Dzubiella, J. Chem. Phys. 143, 064905 (2015).

5. P.D.J. van Oostrum, M. Hejazifar, C. Niedermayer, E. Reimhult, J. Phys.: Condens. Matter 27, 234105 (2015).

6. J.M. Dempster, M.O. de la Cruz, ACS Nano 10, 5909 (2016).

7. M.A. Blanco, V.K. Shen, J. Chem. Phys. 145, 155102 (2016)

8. A.I. Abrikosov, B. Stenqvist, M. Lund, Soft Matter 13, 074902 (2017).

9. M. Sabapathy, R. Ann Mathews K, E. Mani, Phys. Chem. Chem. Phys. 19, 13122 (2017).

10. E. Bianchi, P.D.J. van Oostrum, C.N. Likos, G. Kahl, Curr. Opin. Colloid Interface Sci. 30, 8 (2017).

11. E. Bianchi, B. Capone, I. Coluzza, L. Rovigatti, P.D.J. van Oostrum, Phys. Chem. Chem. Phys. 19, 19847 (2017).

12. M. Stipsitz, E. Bianchi, G. Kahl, J. Chem. Phys. 142, 114905 (2015).

13. E. Bianchi, C.N. Likos, G. Kahl, ACS Nano 7, 4657 (2013).

14. E.G. Noya, I. Kolovos, G. Doppelbauer, G. Kahl, E. Bianchi, Soft Matter 10, 8464 (2014).

15. S. Ferrari, E. Bianchi, Y. Kalyuzhnyi, G. Kahl, J. Phys.: Condens. Matter 27, 234104 (2015).

16. S. Ferrari, E. Bianchi, G. Kahl, Nanoscale 9, 1956 (2017).

17. G. Ciccotti, M. Ferrario, J.P. Rickaert, Mol. Phys. 47, 1253 (1982).

18. W.C. Swope, H.C. Andersen, P.H. Berens, K.R. Wilson, J. Chem. Phys. 76, 637 (1982).

19. H.C. Andersen, J. Comput. Phys. 47, 1253 (1982).

20. L. Angelani, G. Foffi, F. Sciortino, P. Tartaglia, J. Phys.: Condens. Matter 17, L113 (2005).

21. M.P. Allen, D.J. Tildesley, Computer Simulation of Liquids, second edition (Oxford University Press, 2017). 